\title{
Channel-Adaptive Probabilistic Broadcast in Route Discovery Mechanism of MANETs
}

\author{
Haitham Y. Adarbah, and Shakeel Ahmad, Member, IEEE
}

\begin{abstract}
Mobile ad-hoc Networks (MANETs) are selfmanaging wireless networks without relying on any central administration. Each MANET node can connect itself to its neighbors on an ad-hoc basis and communicate with other nodes through its neighbors over multi-hop wireless links. The routes to destination nodes are discovered in an on-demand fashion by broadcasting customized messages called RREQ (Route REQuest). The RREQ messages are re-broadcasted by each of the subsequent receiving nodes until the RREQ arrives at the ultimate destination node which then responds back with the RREP (Route REPly) message to the original sending node to setup up the route. Continuously moving nodes and wireless nature of the communication links impose challenges to efficient broadcasting of RREQs and impact routing performance. A node receiving a RREQ message should be cautious on re-broadcasting it to avoid the Broadcast Storm Problem (BSP) on one side, and on another side, maximizing the reachability of the RREQ message to the destination node. This paper presents a novel distributed algorithm CAPB (Channel Adaptive Probabilistic Broadcasting) to decide the re-broadcasting of RREQ messages for individual MANET nodes in a probabilistic manner. The algorithm takes into account the neighboring node density as well as SINR (Signal to Interference plus Noise Ratio) to decide rebroadcast-probability dynamically. The proposed algorithm has been implemented in the standard AODV routing protocol using the ns-2 simulator. The simulation results have shown that the proposed algorithm outperforms the standard AODV and three state-of-the-art competitor schemes in terms of routing overhead, throughput, end-to-end delay and energy consumption significantly. The proposed algorithm improves network performance and battery life at the same time.
\end{abstract}

Index Terms-MANET; Routing AODV; Probabilistic Broadcast; green computing.

\section{INTRODUCTION}

$\mathrm{T}$ HE ever increasing demand and production of handheld devices (like electronic gadgets, laptops, and smartphones) have made Mobile Ad-hoc Networks a popular

Manuscript received April 6, 2018; revised October 31, 2018. Date of publication February 4, 2019.

Haitham Y. Adarbah is with the Centre for Foundation Studies, Gulf College, Muscat, Oman (haitham.adarbah@gulfcollege.edu.om).

Shakeel Ahmad is with the School of Media Arts \& Technology at Solent University, Southampton SO14 0YN, UK (shakeel.ahmad@solent.ac.uk).

Digital Object Identifier (DOI): 10.24138/jcomss.v15i1.538. choice to communicate with other devices. MANETs have a number of interesting features which gives them an edge over traditional networks e.g., self-configuring and self-healing [1]. These characteristics make MANETs an ideal choice for a number of applications e.g., rescue operations in disaster areas or quick deployment of networks without requiring huge infrastructure like battle fields. These new emerging MANET applications have made MANETs the focal point for many research works. Since, there is no central administration involved in MANETs, node mobility and the wireless nature of multi-hop links make the Quality of Service (QoS) a trembling block in the chain of communications. During the past two decades, improving the QoS has been an active area of research.

The mobility of MANET nodes results in a continuous change of network topology which poses a challenge to stable communication. MANET nodes use the discovery of new neighbors and establish new routes to destination nodes in order to adapt to the changing neighborhood [2]. Nodes have limited transmission range and have to rely on other nodes to relay the message along the route to the final destination node.

A node that wants to transmit a message to a distant node, which is not one of its direct neighbors, first has to trace a set of relay nodes up to the destination node. This is done through the route discovery mechanism. The dynamic nature of the topology due to node movement, limited battery power and the error-prone nature of multi-hop wireless links pose challenges to efficient rout discovery mechanism in MANETs

Routing protocols generally fall into three categories namely table-driven (proactive), on-demand (reactive) and hybrid routing protocols [3][4]. Table-driven routing protocols tend to prepare and maintain routes to all possible destinations in the network at all times. Examples of such protocols include OLSR (Optimized Link State Routing) [5] and DSDV (DestinationSequenced Distance-Vector) routing [6]. On the other side, ondemand protocols discover a route only when it is needed. Examples of on-demand routing protocols include AODV (Adhoc On-demand Distance Vector) routing [7], DSR (Dynamic Source Routing) [3], and ABR (Associativity-Based Routing) [8]. Hybrid routing protocols combine the features of both proactive and reactive routing protocols. Examples of hybrid routing protocols include CEDAR (Core-Extraction Distributed Ad-hoc Routing) [9] and ZRP (Zone Routing Protocol) [10]. 
The interested reader can find a survey in [11].

On-demand routing protocols use two procedures namely route-discovery and route-maintenance to discover and

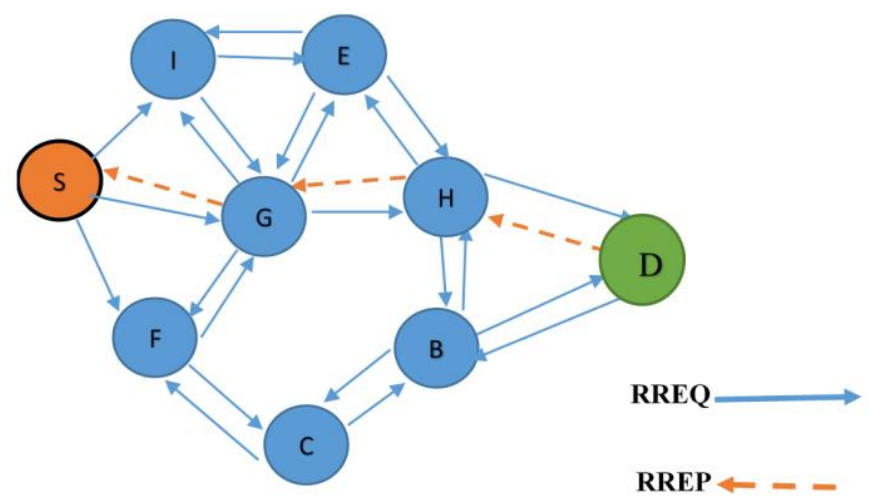

Fig. 1. Example illustration of route discovery in MANETs

maintain routes. Both of these procedures rely on broadcasting to achieve the goals. As an example, in AODV if there is data to be sent to a distant node, a source node uses the route discovery process that starts by broadcasting a special RREQ Packet. The neighboring nodes that receive the broadcasted RREQ packet, forward the received RREQ packet to their neighbors and so on until the RREQ packet is reached at the final destination. The final destination node then sends the RREP packet which follows the reverse path of the RREQ packet to arrive at the destination node. If each node on receiving the RREQ rebroadcasts it once, there would be T-2 rebroadcasts in a network of $\mathrm{T}$ nodes assuming the destination is reachable. This simple mechanism of broadcasting is called pure flooding and is shown graphically in Figure 1 while details can be found in [7].

Pure flooding is a naive scheme and leads to substantial redundant broadcasts because a node often receives the same RREQ packet from more than one neighboring node. This phenomenon leading to a large number of unnecessary rebroadcasts is commonly known as the broadcast storm problem (BSP) in the literature [12]. The BSP is the main source of frequent contention and packet collisions consuming a significant channel bandwidth and increased overhead. This deteriorates network performance in densely populated MANETs. BSP not only affects the route discovery but also affects the route maintenance phase which refreshes the broken routes by triggering new route discovery requests.

Various probabilistic broadcasting schemes were proposed in the literature to address BSP. However, these schemes can be challenged in real life MANETs. This is because real MANETs suffer from noise, co-channel interference and dynamic network topology leading to packet losses, but the schemes proposed in the literature do not consider noise and interference at all $[13,14,15]$ or use simple packet reception model to reflect the effects of noise rather than measuring it at the physical layers [16]. There are multiple channel impairments e.g., signal attenuation, co-channel interference, fading and user mobility that affect wireless communication which must be taken into account. Co-channel interference has been recognized as one of the major factors limiting the capacity of a wireless channel and hence should be considered for realistic performance analysis [17]. This paper presents a novel Channel Adaptive Probabilistic Broadcasting (CAPB) algorithm where the probability of rebroadcasting RREQ packets is adjusted dynamically according to the thermal noise, co-channel interference and node density in the neighborhood. We have implemented the proposed CAPB algorithm in the network simulator ns-2 and ran extensive simulations to compare the proposed algorithm with standard AODV routing protocols as well as three competitor schemes. The performance evaluation has been done using well-known metrics namely routing overhead, throughput, end-to-end delay and energy consumption. Simulation results showed that the proposed algorithm outperforms the standards AODV and the SoA broadcast schemes significantly in all metrics. The proposed algorithm is light weight and does not need any additional information to be exchanged within the neighboring nodes. Parts of this work have been published previously in the two conference papers [18] and [19]. The work of [18] focused on effects of node mobility on the performance of ZigBee networks, while the work of [19] analyzed the effects of node mobility and network size on the performance of MANETs. The work in this paper is a significant extension of the previous work expanding in various dimensions e.g., including more experiments, analysis of rebroadcast probability, effects of traffic on the performance metrics, comparison with more schemes, and analysis of routing traffic.

The rest of the paper is organized as follows: Section II presents the related work, Section III presents the proposed algorithm and Section IV presents simulation results and analysis followed by conclusions in Section V.

\section{RELATED WORK}

Several research works have been proposed in order to elevate the damaging impact of pure flooding [20][21]. In what follows, we review the most recent work related to probabilistic and counter-based broadcasting schemes.

F. Palmieri [22] suggested an adaptive probabilistic approach in which the forwarding probability in an intermediate node is adjusted according to the distance of the sender. Their ns-2 simulation results showed that the new approach, when applied to AODV, enhanced the network performance in terms of routing overhead, throughput, and latency. Q. Jiang and D. Manivannan [23] proposed the TBR algorithm that reduces the redundant rebroadcasting of RREQ messages during route discovery. The TBR divides the network topology into triangular regions and RREQ messages are forwarded based on regions which have already been visited. The TBR algorithm was shown to improve performance over the standard AODV protocol in terms of packet delivery ratio, end-to-end delay, and routing overhead. M. B. Khalaf, et al. [24] suggested two probabilistic route discovery schemes called SVAP (Simple Velocity Aware Probabilistic) and AVAP (Advanced Velocity Aware Probabilistic). These schemes consider node velocities into account. Nodes with the same velocities are more likely to forward than nodes with different velocities. Zhang and Zhou [13] proposed an algorithm of load balancing based on history 

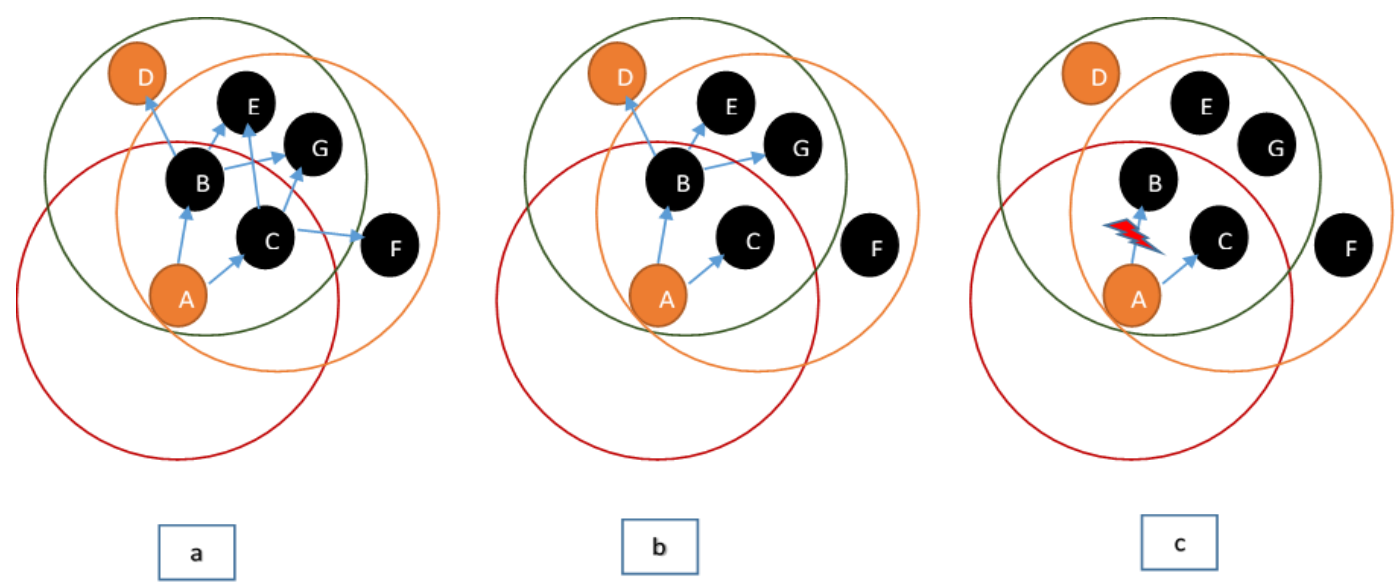

Fig. 2. (a) Pure flooding in MANETs in noiseless MANETs (b) Static Probabilistic scheme in noiseless MANETs (c) Static Probabilistic scheme in noisy MANETs

information. In their algorithm, mobile nodes use history information and judge route access probabilistically. The work,

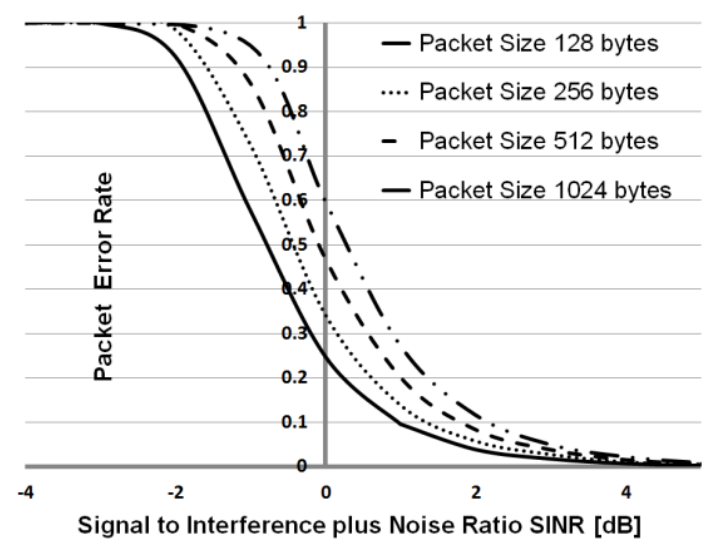

Fig. 3. PER (Packet Error Rate) and SINR relationship for different packet sizes [29].

they named HPDSR (Historical Probability Dynamic Source Routing) was implemented in standard DSR (Dynamic Source Routing) and showed performance improvement over standard DSR through simulation. Ali, et al. [25] proposed a neighborhood-based routing protocol called DCFP (Dynamic Connectivity Factor routing Protocol) that dynamically probes the status of the underlying network without the intervention of a system administrator while reducing the RREQ overhead using a new connectivity factor. The results of DCFP revealed that the suggested protocol showed better performance than AODV in terms of end-to-end delay, normalized routing overhead, MAC collision, energy consumption, network connectivity, and packet delivery ratio.

A slightly different category of broadcasting schemes is counter based schemes. In counter-based schemes, a node counts the number of duplicate broadcast packets it receives during a time period called Random Assessment Delay. Then the number of duplicates is compared to a threshold value to decide whether to re-broadcast or not [14]. Bani Yassein et. al. [26] suggested a dynamic counter based scheme where the threshold of duplicate packets is adjusted dynamically depending on the number of nodes around.

The authors of [27] present a dynamic probabilistic scheme where the probability of re-broadcasting changes with the node density. Only a few schemes considered the noise e.g., the work of [16] where the probability adapts to the noise level dynamically. However, the work used a distribution to infer packet reception as a result of noise rather than measuring the actual noise level at the physical layer.

To the best of our knowledge, no previous work has considered the effects of real thermal noise, co-channel interference and the node density simultaneously to address the broadcast storm problem in the route discovery process of MANETs, which is the novelty of our work.

\section{PROPOSED BRoAdCAST SchEME}

In the proposed CAPB algorithm, when a MANET node receives a RREQ packets, it determines the rebroadcast probability dynamically on the fly by taking into account two variables. The first variable is the measured co-channel interference plus thermal noise and the second variable is the number of nodes in the vicinity which are likely to have received the same RREQ packet and are considering to rebroadcast. In the following, the two variables are discussed in detail how they would affect the BSP and successful delivery of the RREQ packet to the destination.

\section{A. Effect of Co-Channel Interference \& Thermal Noise}

Consider Figure 2 where node A broadcasts a RREQ message to find a route to node G. In Figure 2(a), using pure flooding in absence of co-channel interference and thermal noise, the destination node $(G)$ receives the RREQ packet from node $\mathrm{B}$ as well as node $\mathrm{C}$. The node $\mathrm{G}$ however, will only send one RREP packet to either node $\mathrm{B}$ or $\mathrm{C}$ whichever forwards the RREQ first. Using probabilistic broadcast, there are three possibilities (i) both B and C, (ii) either B or C and (iii) neither of the two nodes will rebroadcast the RREQ packet. As exemplified in Figure $2 \mathrm{~b}$, using probabilistic broadcast in absence of co-channel interference and thermal noise, only node $\mathrm{B}$ manages to rebroadcast the RREQ. By considering the effects of thermal noise and co-channel interference (Figure 2c), assuming that node A fails to deliver the RREQ packet to node $\mathrm{B}$ (because of thermal noise plus interference in the area), but is able to deliver the same packet to node C, the RREQ packet is therefore undelivered to node $G$. Node $G$ will thus be 


\section{Event: Node $R$ receives RREQ packet $m$ \\ if Node $R$ is the destination node for RREQ $m$ Send RREP}

else

Calculate $N_{b}$

Measure SINR and calculate PER

Calculate $N_{\text {eff }}$ using eq. 4

Calculate $P_{\text {reb }}$ from eq. 6

Generate a random number $\boldsymbol{\delta}$ between 0 and 1.0

if $\boldsymbol{\delta}<=P_{r e b}$ then

Broadcast the RREQ message $m$

else

Drop the RREQ message $m$

end if

end if

End if

Fig. 4. Proposed CAPB algorithm

declared unreachable.

Packet Error Rate (PER) is closely related to SINR (Signal to Interference plus Noise Ratio) and packet size as shown in Figure 3. In the proposed CAPB algorithm, when a node receives a RREQ packet, it obtains the SINR value, as measured at the physical layer and infers the corresponding PER using the relationship shown in Figure 3. If the PER is higher, then the probability of receiving the same RREQ packet by the neighboring nodes is low. In this case, naturally, the lucky node that has received the RREQ should rebroadcast the RREQ with high probability to increase the dissemination of this particular RREQ packet. On the other hand, a low PER implies that many nodes in the neighborhood have also received the RREQ packet with high probability, therefore the rebroadcast probability should be relatively low to avoid the BSP.

\section{B. Effect of Neighborhood Density}

When a node receives a RREQ packet, the decision of rebroadcasting should take into account the number of neighboring nodes and their geographic distribution to make a wise decision. In a densely populated area, not all nodes need to rebroadcast to avoid redundancy and the risk of increased collision leading to packet loss and energy wastage. However, in a sparsely populated area relatively more nodes should rebroadcast the RREQ packet to ensure dissemination of the RREQ packet. Here we consider only the number of nodes in the transmission range of the node receiving the RREQ packet to determine the rebroadcast probability.

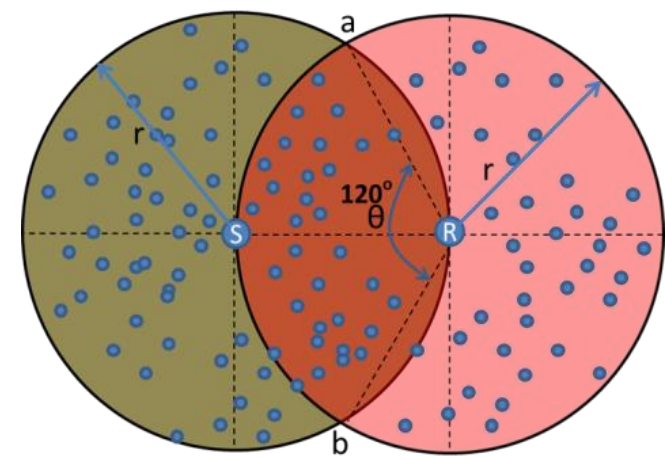

Fig. 5. Node R receiving RREQ from node $S$.

\section{The Proposed CAPB Algorithm}

Figure 4 shows the proposed CAPB algorithm. Let's consider the event when node $R$ receives RREQ message $m$ from node $\mathrm{S}$. If node $\mathrm{R}$ is not the destination node, it would rebroadcast the received packet $m$ with probability $P_{r e b}$. To determine the value of $P_{r e b}$, node R first determines the value of $N_{e f f}$. Here $N_{e f f}$ denotes the number of effective nodes lying within node R's transmission range $r$ which might have received the same RREQ packet $m$. Node R will use Hello packets to infer the total number of nodes $\mathrm{N}$ within its transmission range $\mathrm{r}$. Then the number of nodes $N_{b}$ which are located within the transmission range of both Node $S$ and $R$ can be calculated from N. As shown in Figure 5, the value of $N_{b}$ is effectively the overlapped area A of the two circles. Using geometry, it can be shown that the overlapped area $\mathrm{A}$ is given by the following equation.

$$
A=\left(\theta \times \frac{\pi}{180}-\sin (\theta)\right) \times r^{2}
$$

The angle $\theta$ in equation (1) is the angle of the circular segment in degrees as shown in Figure 5. When node $\mathrm{R}$ is at the edge of the transmission range $r$ of node $S$, then $\theta=120^{\circ}$ and when node $\mathrm{R}$ and $\mathrm{S}$ are co-located then $\theta=180^{\circ}$.

Node $\mathrm{R}$ can estimate its distance from node $\mathrm{S}$ by measuring the signal strength of the received RREQ packet. Note that the distance estimation may be slightly inaccurate due to the added noise and co-channel interference. The proposed algorithm relies on the approximate distance estimation rather than the exact distance in order to keep the complexity low. The estimated approximate distance is then used to calculate the value of $\theta$ using simple trigonometric relations as shown in Figure 5. Assuming that the nodes are uniformly distributed, the value of $\mathrm{N}_{\mathrm{b}}$ can be given by

$$
N_{b}=N \times A / \pi r^{2}
$$

Node R measures the SINR from the physical layer at the time of receiving the RREQ packet $m$ and computes the PER using the relationship shown in Figure 3. The value of $N_{e f f}$ is then given by

$$
N_{e f f}=N_{b} \times(1-P E R)
$$


Equation (3) can be simplified to

$$
N_{e f f}=N \times\left(\frac{\theta}{180}-\frac{\sin (\theta)}{\pi}\right)(1-P E R)
$$

A higher value of $N_{\text {eff }}$ means that a higher number of nodes might have received the same RREQ packet $m$ and thus the value of $P_{r e b}$ should be lower and vice versa. This shows an inverse relationship between $P_{r e b}$ and $N_{e f f}$.

$$
P_{r e b}=d \times \frac{1}{N_{e f f}}
$$

Here $d$ is a constant value which can be tuned to maximize performance. For very low $\left(\leq N_{l}\right)$ and very high $\left(\geq N_{u}\right)$ values of $N_{\text {eff }}$ equation (5) does not hold and fixed values of $P_{r e b}$ should be used instead. In general, $P_{r e b}$ can be given as follows:

$$
P_{r e b}=\left\{\begin{array}{lr}
P_{\text {max }}, & \text { for } N_{e f f} \leq N_{l} \\
d \times \frac{1}{N_{e f f}}, & \text { for } N_{l}<N_{e f f}<N_{u} \\
P_{\text {min }}, & \text { for } N_{e f f} \geq N_{u}
\end{array}\right.
$$

An estimated maximum and minimum possible node density and transmission range of nodes can be used to set appropriate values of $N_{l}$ and $N_{u}$. The next section shows the implementation and evaluation of the proposed algorithm.

\section{Performance Evaluation of The CAPB Algorithm}

This section presents the performance evaluation of the proposed CAPB algorithm using four metrics namely routing overhead, throughput, end-to-end delay and energy consumption for different node densities, mobility profiles, and traffic load. The traffic load is varied by varying number of source-destination connections. The proposed CAPB algorithm has been compared with four other broadcasting schemes. The first one is standard AODV routing protocol, the second one is the fixed probabilistic scheme [12] denoted by AODV-P where $P$ shows the rebroadcast probability, the third scheme denoted by AODV-DNDP is dynamic noise-dependent probabilistic scheme of [16], and the fourth one denoted by AODV-DCB is dynamic counter based scheme of [26].

\section{A. Simulation Setup}

In order to do the implementation and evaluation of the proposed algorithm in MANETs we used ns2 simulator (2.35v). The proposed CAPB algorithm and the three competitor schemes namely AODV-P (fixed probability) [12], AODVDNDP (Dynamic Noise Dependent Probabilistic) [16], and AODV-DCB (Dynamic Counter Based) [26] have been implemented in the route discovery process of AODV. In AODV-P [12] the RREQ packet is rebroadcasted with a fixed probability $\mathrm{P}$. We set the value of $\mathrm{P}$ after simulating a range of values for $\mathrm{P}$, and chose the value that gave the best performance.

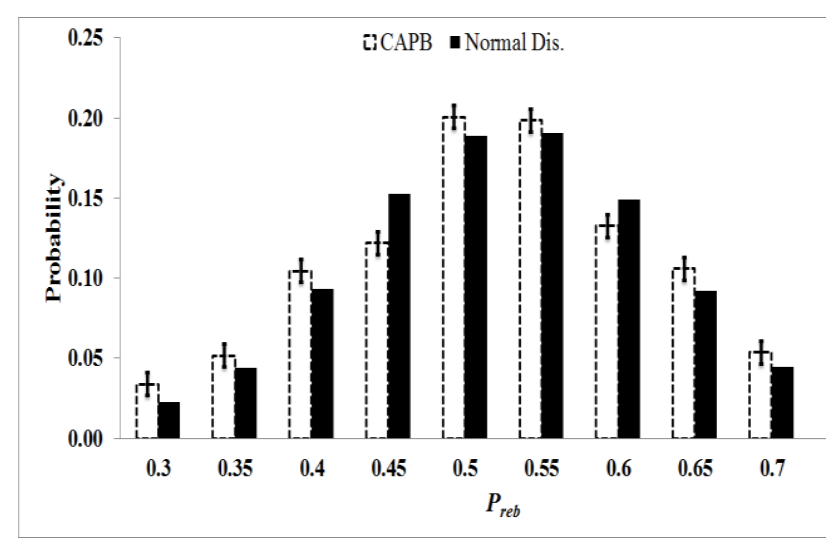

Fig. 6. Probability distribution function of $P_{r e b}$

The parameters of AODV-DNDP and AODV-DCB follow recommendations of [16] and [26] respectively. For CAPB, we set $N_{l}=7, N_{u}=16, P_{\max }=0.7, P_{\min }=0.3$ and $\mathrm{d}=5$. These values are partly heuristic and partly simulation guided.

The MANET related simulation parameters generally follow [16][28]. The radio propagation is based on 2-ray Ground Reflected Model. The network bandwidth is set to $6 \mathrm{Mbps}$ and the medium access control (MAC) protocol is simulated using the ns-2 library dei80211mr [29]. This library calculates the PER using pre-determined curves (PER Vs. SINR) for the given packet size (shown in Figure 3). The SINR value is computed from the received signal strength, thermal noise, and co-channel interference. Thermal noise is set to $-95 \mathrm{dBm}$ following recommendations in [30].

We used Random Waypoint [31] mobility model, and we set variable node speed and pause time set to zero in order to model the node mobility. Nodes are placed randomly in an area of $1000 \times 1000$ square meters. Transmission power, path loss and receive power threshold are set such that the effective transmission range is $250 \mathrm{~m}$. Each node has an FTP (File Transfer Protocol) agent attached to it such that node $i$ is downloading a file of infinite size from node $\mathrm{i}+\mathrm{M} / 2$ for $\mathrm{i}=1,2, \ldots, \mathrm{M} / 2$ where $\mathrm{M}$ is the total number of nodes for density and mobility scenarios. Each node was set an initial energy of 1000 joules for analyzing energy consumption.

\section{B. Simulation Results and Analysis}

We used three different simulation scenarios namely the density-scenario, the mobility-scenario, and the traffic-scenario to see effects of varying node density, mobility, and traffic load respectively on the performance metrics (routing overhead, throughput, end-to-end delay and energy consumption). There are three variables namely mobility, number of nodes, and traffic involved in the three scenarios. In each scenario, one variable is varied while the other two variables are fixed. The density and traffic load scenarios use a fixed node speed of $6 \mathrm{~km} /$ hour for each node. The mobility and the traffic load scenarios use fixed number of nodes (set to 100). Similarly, the density and mobility scenarios use fixed number of sourcedestination connections which is set to 50 . 


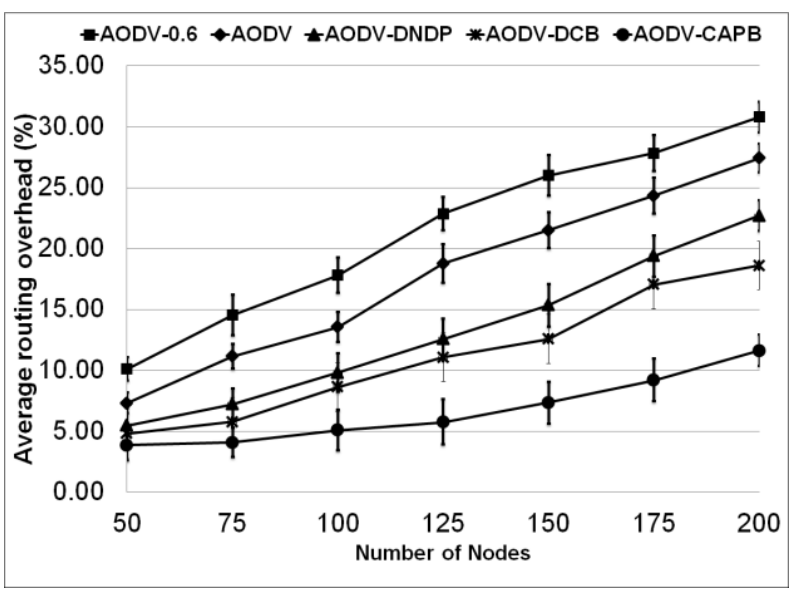

Fig. 7. Routing Overhead vs Number of Nodes (density-scenario)

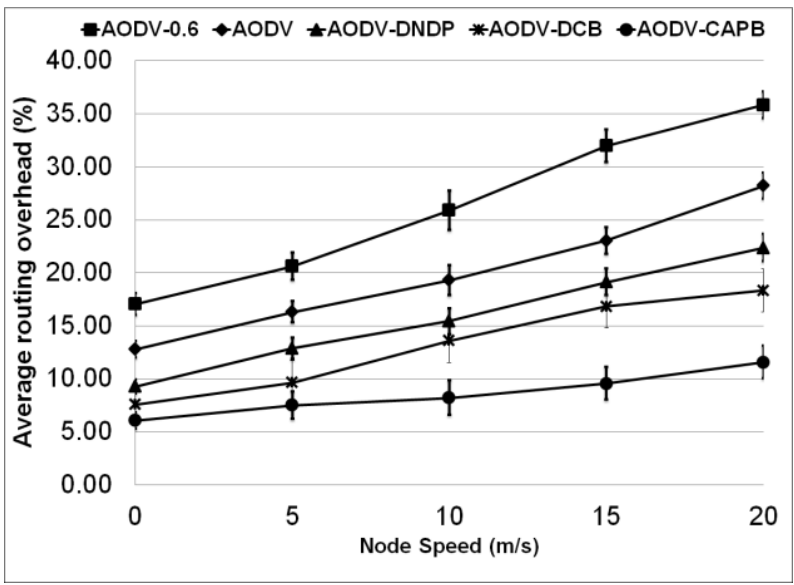

Fig. 8. Routing Overhead vs Node Speed (mobility-scenario)

To obtain simulation results, we got the averaged the results of 30 runs for each scenario. All scenarios had the same confidence interval of $95 \%$. Also, each scenario used a different seed value, and the simulation time was set to 800 seconds. The seed value is used in the mobility model to yield different mobility profiles and to set the initial location for each node. Since the direct outcome of the proposed CAPB algorithm is the probability $\mathrm{P}_{\mathrm{reb}}$ of rebroadcasting RREQ, it would be worth seeing statistical properties of $\mathrm{P}_{\mathrm{reb}}$. We collected $\mathrm{P}_{\text {reb }}$ values from all the simulation runs for all scenarios and plotted the histogram of $\mathrm{P}_{\text {reb }}$ values as shown in Figure 6. The mean and variance of $\mathrm{P}_{\mathrm{reb}}$ is found to be 0.5 and 0.01 respectively. It is interesting to observe that the distribution of $\mathrm{P}_{\text {reb }}$ follows closely the normal distribution truncated at just below 0.3 and above 0.7 with the same mean and deviation. From the derivation of the proposed algorithm, it can be seen that the value of $\mathrm{P}_{\mathrm{reb}}$ depends on two random variables, the noise level (thermal noise plus co-channel interference) which leads to PER, and the number of nodes in the neighbourhood. Note that co-channel interference is log-normal and thermal noise is normal distributed [17]. Since nodes are moving randomly, using central limit theorem, it can be shown that the number of

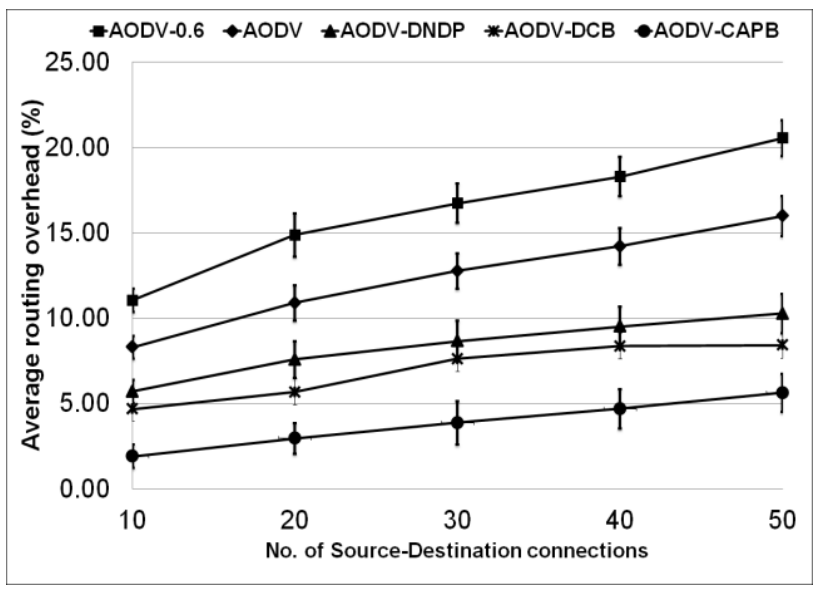

Fig. 9. Routing Overhead vs traffic load (traffic-scenario)

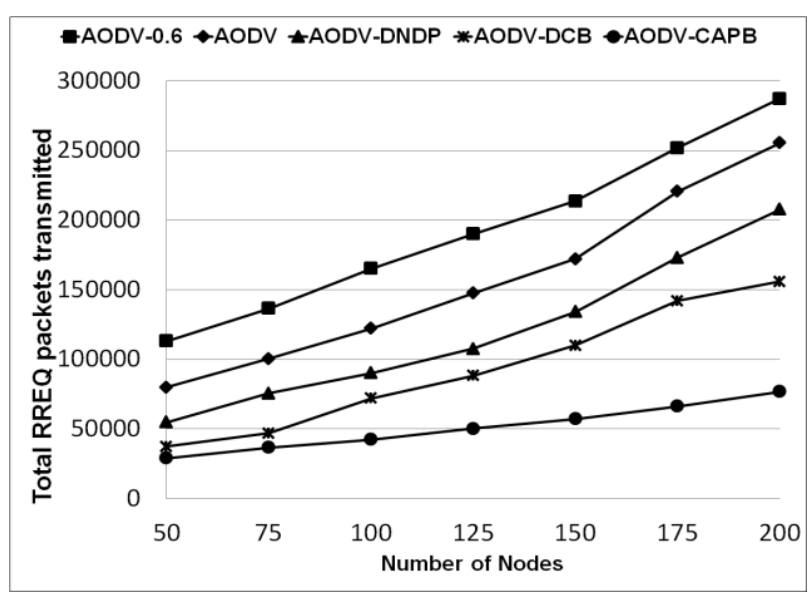

Fig. 10. Total number of RREQ packets transmitted for different number of nodes (density-scenario)

nodes in the neighbourhood would have a normal distribution. This explains why $\mathrm{P}_{\mathrm{reb}}$ is normally distributed.

\section{1) Routing Overhead}

Routing overhead is defined as the ratio of the number of routing packets (control packets) transmitted per data packet received. Figure 7, Figure 8 and Figure 9 show the average routing overhead as a function of node density, node speed, and traffic load respectively.

In general, the average routing overhead increases with increasing node density and traffic load because a higher number of neighboring nodes and traffic load lead to higher contention and PER which result in redundant retransmission of control packets. Similarly, increasing node speed makes the network topology more dynamic, routes get expired quickly and new route discovery mechanism is triggered more often to replace the expired routes. This can be verified by observing the total number of RREQ packets transmitted as shown in Figure 10, Figure 11 and Figure 12.

The proposed CAPB algorithm uses the lowest number of RREQ packets. Increasing the number of RREQ broadcasts increases the reachability of nodes on one hand but on another 


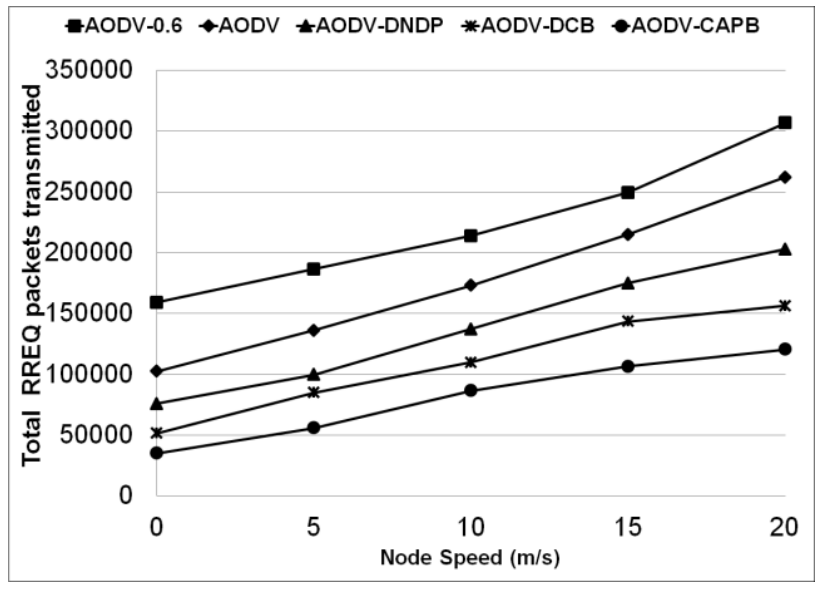

Fig. 11. Total number of RREQ packets transmitted (mobility-scenario)

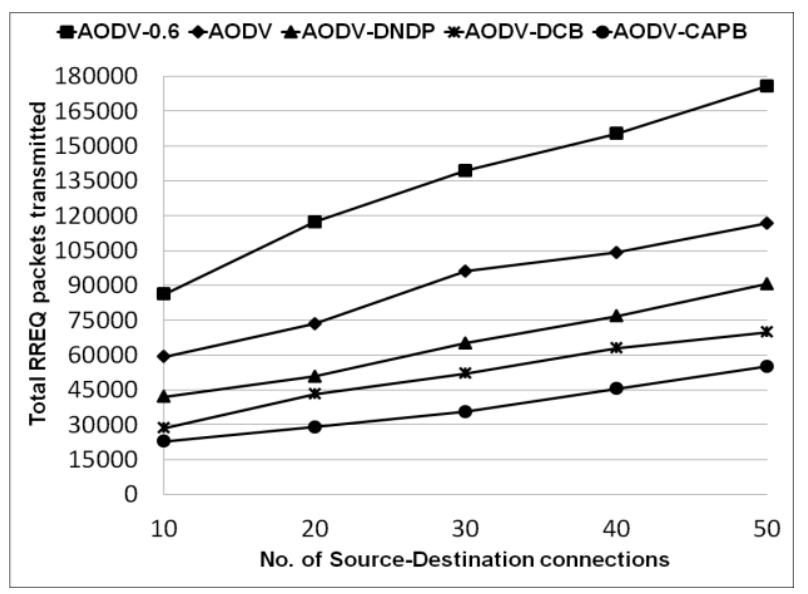

Fig. 12. Total number of RREQ packets transmitted vs. traffic load (trafficscenario)

hand, it may increase the co-channel interference leading to higher PER which may limit the reachability and require to restart the route discovery process. This is the reason for the higher overhead of pure AODV scheme. Fixed probabilistic scheme (AODV-0.6) limits the number of RREQ blindly which often limits the reachability of RREQ packets to the destination node and the route discovery mechanism has to be triggered more often leading to higher overhead. It is interesting to note that the routing overhead of pure AODV is better than AODV0.6 scheme. In fact, thermal noise plus co-channel interference act as natural limiters for the traffic; the former is static while the latter is adaptive because it increases with traffic intensity. This reduces the chances of getting duplicate RREQs from the neighboring nodes and adapts to the traffic intensity very well.

In presence of natural and adaptive limiting factor (thermal noise and co-channel interference), the artificial limiter (i.e., reducing the rebroadcast probability without considering the effect of interference and thermal noise) does not work well because it limits the reachability of RREQs independent of the traffic intensity. Nodes have to try several times before they get a valid route which increases the routing overhead. In both AODV-DNDP and AODV-DCB, the probability is not fixed and is drawn from a distribution and the three counter

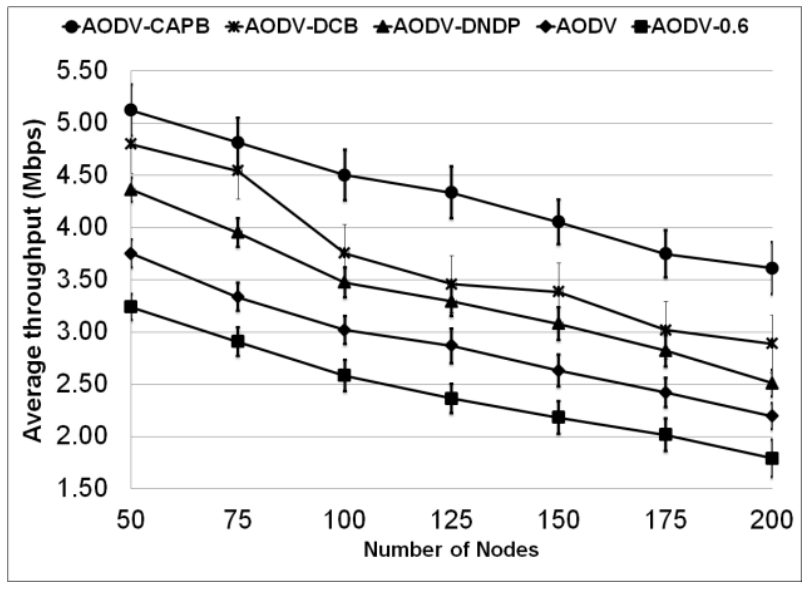

Fig. 13 Average throughput vs. Number of Nodes (density-scenario)

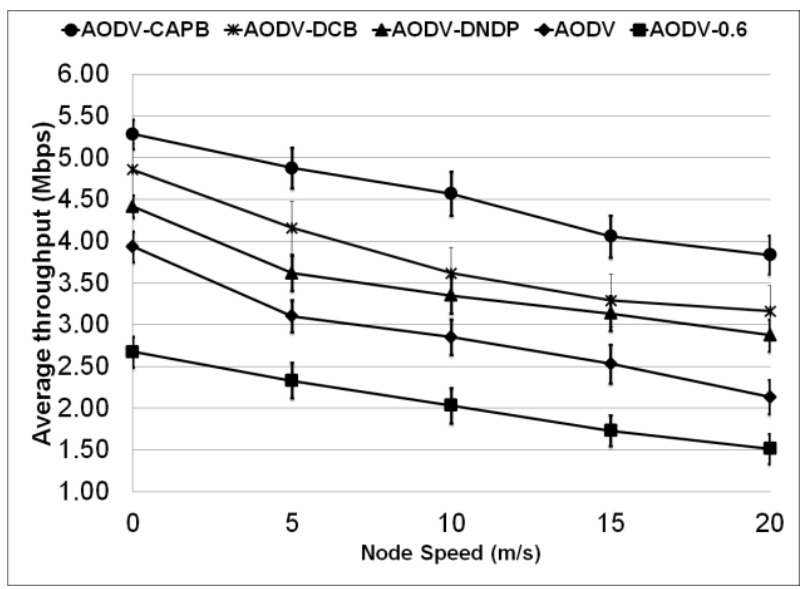

Fig. 14. Average throughput vs. Node Speed (mobility-scenario)

thresholds are assigned without considering the current level of noise and interference. The proposed CAPB algorithm is able to achieve significantly lower routing overhead as compared to other schemes as shown in Figure 7, 8 and 9. The comparative savings in routing overhead increases with the increase in node density, node speed, and traffic load.

\section{2) Average Throughput}

Throughput is defined as the amount of data received by a node per unit time. Figure 13 shows the throughput, measured at the application layer, averaged for all nodes as a function of a number of nodes. Figure 14 shows the average throughput as a function of node speed and Figure 15 shows the average throughput as a function of traffic load.

As a general trend, the average throughput of nodes goes down with increasing number of nodes. This is because of increased contention and collision among the nodes when they try to transmit which in turns limits the achievable throughput. The average throughput also suffers from increased node speed. This is because increased speed changes the topology more often leading to broken routes causing a temporary pause in data transmission untill a new route is found.

Rebroadcasting the RREQ packets blindly without taking into account other factors may not lead to finding the route at 


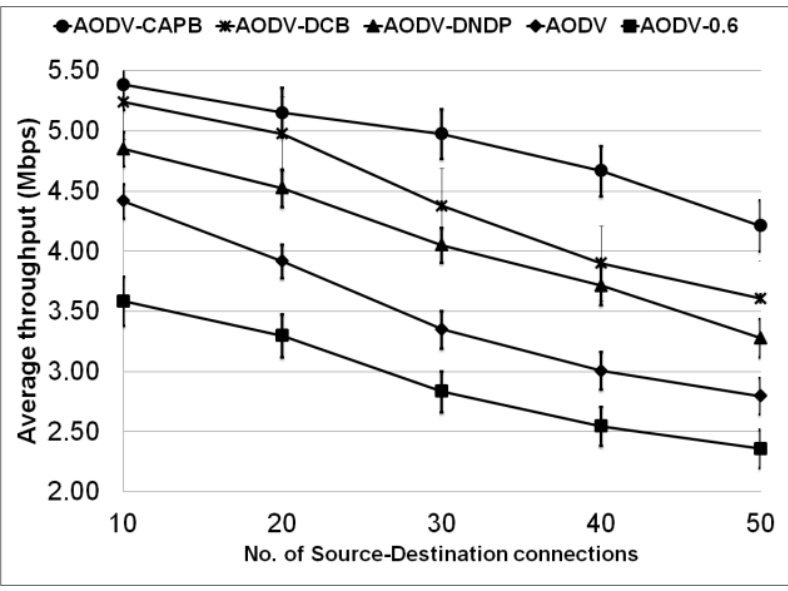

Fig. 15. Average throughput vs. traffic load (traffic-scenario)

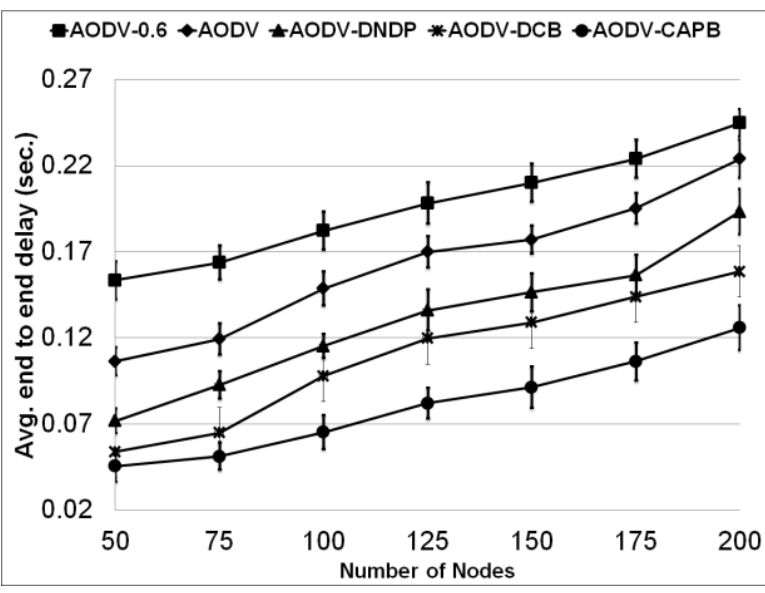

Fig. 16. Average end to end delay vs. number of node (density-scenario)

first attempt. The route discovery process might need to be triggered several times which means longer time to establish the route. This also means that the FTP application has to wait longer to resume the transmission. The proposed algorithm is able to enhance the throughput significantly over the other competitor schemes. This is because the rebroadcasting decision in CAPB is better informed by SINR and nodal density in the neighborhood which increases the reachability of RREQ to the destination node while keeping the routing overhead at a minimum.

\section{3) Average End-to-End Delay}

The average end-to-end delay is referred to the packet travel time between the source and the destination. It includes all possible delays which include buffering during route discovery, queuing at the interface queue, retransmission delays at the MAC, propagation delay and transmission delay. Figure 16, 17 and 18 show the average end-to-end delay for data packets for all nodes as a function of a number of nodes, as a function of node speed and as a function of traffic load respectively.

It can be seen that for all schemes, the average end-to-end delay increases with increasing number of nodes, node speed, and traffic load. By increasing the number of node and traffic load, contention increases leading to higher queuing delay at the transmitter's buffer and higher packet loss rate due to the

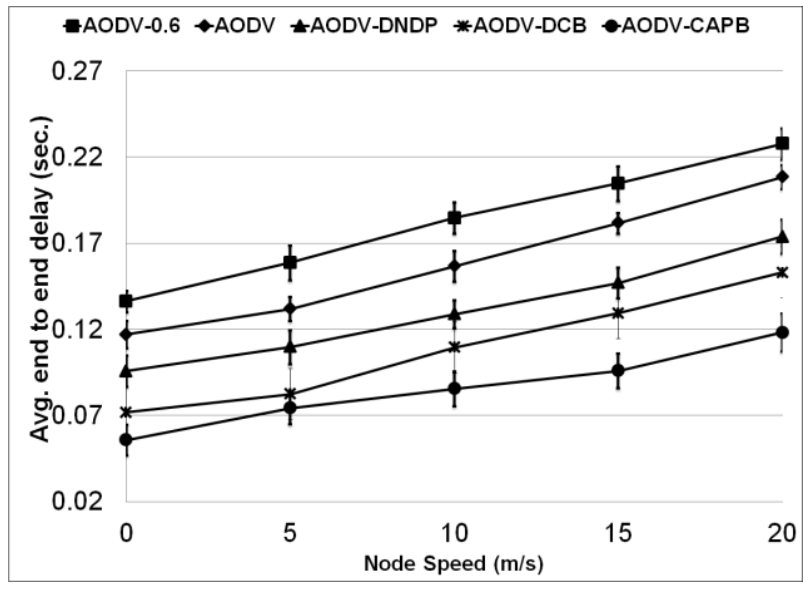

Fig. 17. Average end to end delay vs. node speed (mobility-scenario)

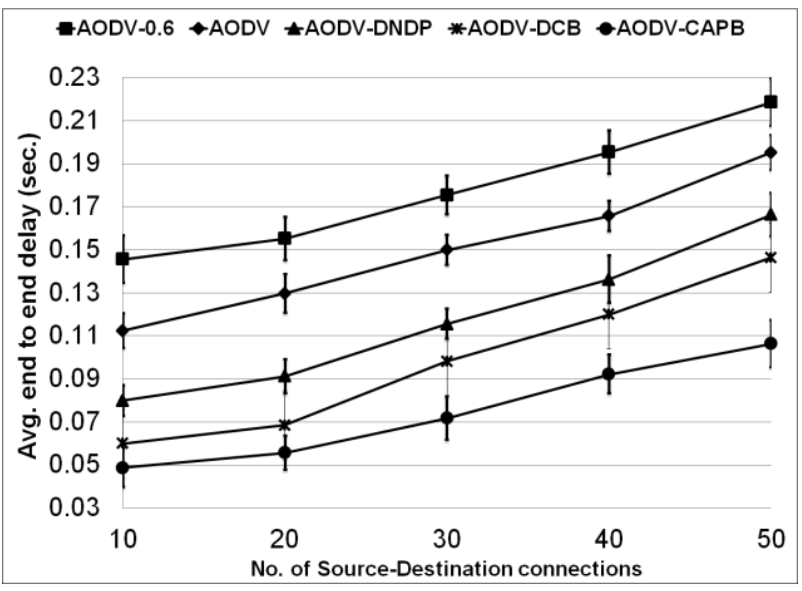

Fig. 18. Average end to end delay vs. traffic load (traffic-scenario)

increased collision. A data packet may need to be retransmitted multiple times. With increased mobility, route breaking and repairing take places more often leading to a higher average delay.

The proposed CAPB algorithm achieves much lower end-toend delay as compared to other schemes. This is because the proposed scheme produces fewer routing traffic, which helps to decrease the contention and collision, and it increases the reachability of RREQ packets to the destination which helps to establish or repair routes faster.

\section{4) Average Energy Consumption}

Energy consumption is referred to the amount of energy spent on transmitting, forwarding and receiving packets (both data and routing packets). We used the energy model in ns-2 to measure the energy consumption of AODV, AODV-0.6, AODV-DNDP, AODV-DCB, and AODV-CAPB. As implemented in ns-2, an energy model is a node attribute and it represents energy level in a mobile node. It has an initial value which is the level of energy the node has at the beginning of the simulation and also has a given energy usage for every packet it transmits and receives. These are txPower_and rxPower, and we used the default value for them which is $281.8 \mathrm{~mW}$ [29].

Figure 19, Figure 20 and Figure 21 depict the average energy consumption for all nodes for the three scenarios. The proposed 


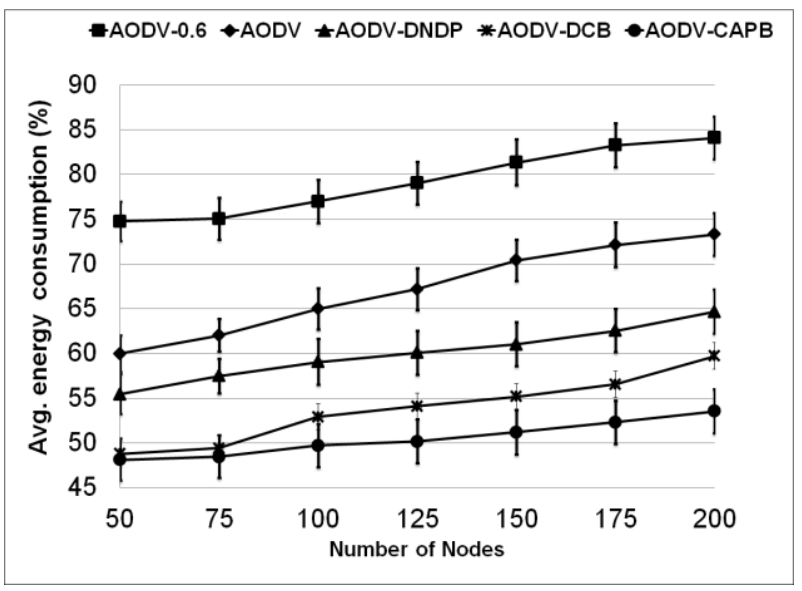

Fig.19. Energy Consumption vs. Number of Nodes (density-scenario)

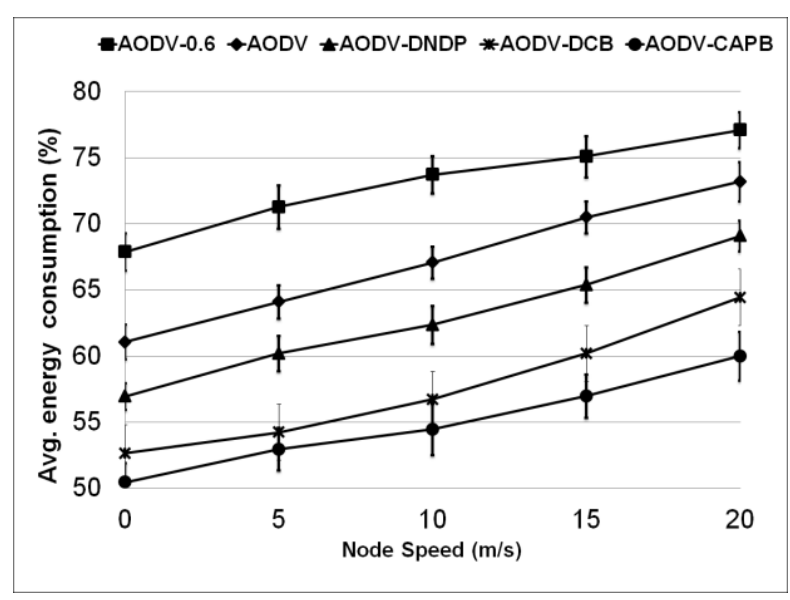

Fig.20. Energy Consumption vs. Node Speed (mobility-scenario)

CAPB algorithm achieves better energy efficiency as compared to the other schemes. The energy saving of CAPB is achieved by adapting the rebroadcasting of RREQ packets to current channel conditions and the number of neighboring nodes which helps to reduce unnecessary transmissions of RREQ packet.

However, the savings in energy is not in proportion to the saving in RREQ packets (see Figure 7, Figure 8 and Figure 9). This is because the CAPB algorithm achieves much higher throughput as well which consumes extra energy.

\section{CONCLUSION AND FUTURE WORK}

On-demand routing protocols in MANET rely on broadcasting to discover and then maintain routes. Standard (e.g., AODV) uses pure flooding to broadcast the RREQ packets. However, pure flooding generates excessive control traffic which may lead to the broadcast storm problem. A number of broadcasting schemes have been proposed in the literature to limit the broadcast traffic, but these schemes do not consider thermal noise and co-channel interference and hence do not perform well in realistic noisy MANETs. Node density in the neighborhood is another important factor to determine the rebroadcast probability. This paper has presented a novel Channel Adaptive Probabilistic Broadcast (CAPB) algorithm

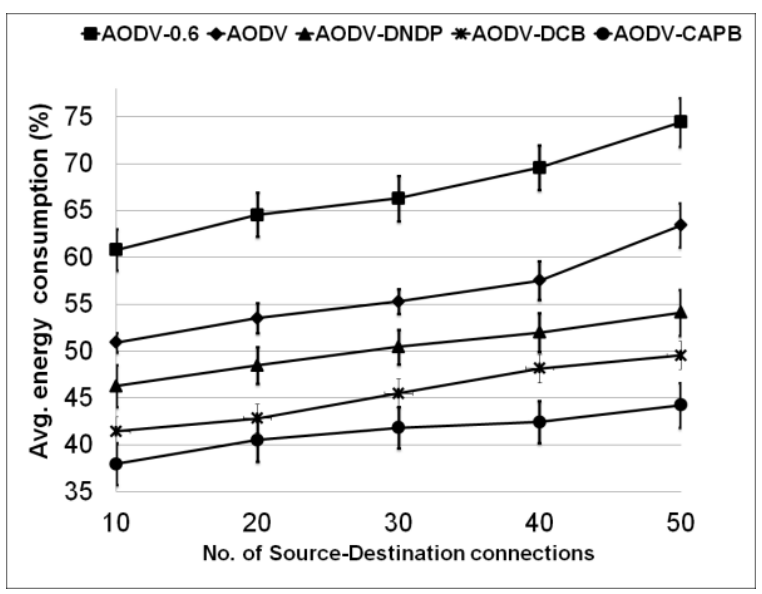

Fig.21. Energy Consumption vs. traffic load (traffic-scenario)

that adapts the rebroadcast probability to the thermal noise, cochannel interference and node density in the neighborhood dynamically. Extensive ns-2 simulations have shown that the proposed CAPB algorithm outperforms the standard AODV and the three related schemes significantly in terms of routing overhead, throughput, end-to-end delay and energy consumption. Simulation results also revealed that the distribution of the rebroadcast probability follows normal distribution closely. The proposed algorithm is simple and does not require any extra information to be exchanged among the neighboring nodes.

The proposed algorithm shows the potential gains of considering thermal noise, co-channel interference, and node density in the neighborhood. However, the proposed algorithm depends on carefully chosen values of certain parameters $\left(N_{l}\right.$, $N_{u}, P_{\max }, P_{\min }$ and $\left.d\right)$. These parameters were chosen partly heuristically and partly simulation guided in this work. However, research on a systematic approach to find out the optimal values of the aforementioned parameters would be a potential extension of this work.

\section{REFERENCES}

[1] J. Liu and X. Jiang, "Throughput Capacity of MANETs with Power Control and Packet Redundancy," IEEE Trans. Wirel. Commun., vol. 12, no. 6, pp. 3035-3047, 2013. DOI: 10.1109/TWC.2013.042413.121683.

[2] C. Ni, H. Liu, A. G. Bourgeois, and Y. Pan, "An enhanced approach to determine connected dominating sets for routing in mobile ad hoc networks," Int. J. Mob. Commun., vol. 3, no. 3, pp. 287-302, 2005. DOI: 10.1504/IJMC.2005.006585.

[3] N. Sarkar and W. Lol, "A study of manet routing protocols: Joint node density, packet length and mobility," in IEEE Symposium on Computers and Communications (ISCC), 2010, pp. 515-520. DOI: 10.1109/ISCC.2010.5546763.

[4] M. Abolhasan, "A review of routing protocols for mobile ad hoc networks," Ad Hoc Networks, vol. 2, no. 1, pp. 1-22, Jan. 2004. doi.org/10.1016/S1570-8705(03)00043-X.

[5] T. Clausen and P. Jacquet, "Optimized Link State Routing Protocol (OLSR)," RFC 3626, IETF Network Working Group, 2003. [Online]. Available: http://www.ietf.org/rfc/rfc3626.txt. [Accessed: 03-Apr-2018].

[6] C. E. Perkins and P. Bhagwat, "Highly Dynamic ( DSDV ) for Mobile Computers Routing," in In Proceedings of the SIGCOMM '94 Conference on Communications Architectures, Protocols and Applications, 1994, pp. 234-244. DOI:10.1145/190314.190336.

[7] S.-J. Lee, E. M. Belding-Royer, and C. E. Perkins, "Ad hoc on-demand distance-vector routing scalability," ACM SIGMOBILE Mob. Comput. Commun. Rev., vol. 6, no. 3, p. 94, Jun. 2002. DOI: 
$10.1145 / 581291.581306$

[8] C. K. Toh, "Associativity-Based Routing for Ad Hoc Mobile Networks," Wirel. Pers. Commun., vol. 4, no. 2, pp. 1-36, 1997. doi.org/10.1023/A:1008812928561.

[9] P. Sinha, R. Sivakumar, and V. Bahrghavan, "CEDAR: A Core Extraction Distributed Ad Hoc Roouting Algorithm," IEEE J. Sel. Areas Commun., vol. 17, no. 8, pp. 1454-1466, 1999. DOI 10.1109/INFCOM.1999.749269.

[10] Z. J. Haas and M. R. Pearlman, "The performance of query control schemes for the zone routing protocol," in in Proc. ACM SIGCOMM '98, 1998, pp. 167-177. DOI:10.1145/285243.285279

[11] A. Boukerche, B. Turgut, N. Aydin, M. Z. Ahmad, L. Bölöni, and D. Turgut, "Routing protocols in ad hoc networks: A survey," Comput. Networks, vol. 55, no. 13, pp. 3032-3080, 2011 doi.org/10.1016/j.comnet.2011.05.010.

[12] S. Ni, Y. Tseng, and Y. Chen, "The broadcast storm problem in a mobile ad hoc network," Wirel. Networks, no. 8, pp. 153-167, 2002 doi.org/10.1023/A:1013763825347.

[13] D. Zhang and D. Zhou, "Load Balancing Algorithm Based on History Information In MANET," in 2017 IEEE 2nd Information Technology, Networking, Electronic and Automation Control Conference (ITNEC), 2017, pp. 737-742. DOI: 10.1109/ITNEC.2017.8284830.

[14] A. Mohammed, M. Ould-Khaoua, L. M. Mackenzie, C. Perkins, and J.-D. Abdulai, "Probabilistic counter-based route discovery for mobile ad hoc networks," in Proceedings of the 2009 International Conference on Wireless Communications and Mobile Computing Connecting the World Wirelessly - IWCMC '09, 2009, pp. 1335-1339. DOI $10.1145 / 1582379.1582672$

[15] J. Cartigny and D. Simplot, "Border Node Retransmission Based Probabilistic Broadcast Protocols in Ad-Hoc," in The 36th Annual Hawaii International Conference on System Sciences (HICSS'03), 2003, pp. 6-9. DOI: 10.1109/HICSS.2003.1174853.

[16] H. Al-Bahadili and A. Sabri, "A Novel Dynamic Noise-Dependen Probabilistic Algorithm for Route Discovery in MANETs," Int. J. Bus. Data Commun. Netw., vol. 7, no. 1, pp. 52-67, 2011. DOI: 10.4018/jbden.2011010103.

[17] P. Cardieri and T. S. Rappaport, "Statistical analysis of co-channel interference in wireless communications systems," Wirel. Commun. Mob. Comput., vol. 1, no. 1, pp. 111-121, 2001. doi.org/10.1002/1530 8677(200101/03)1:1<111::AID WCM5>3.0.CO;2-5.

[18] H. Y. Adarbah and S. Ahmad, "Efficient route discovery using channel adaptive probabilistic broadcasting in Zigbee wireless sensor networks," in 2017 25th International Conference on Software, Telecommunications and Computer Networks, SoftCOM 2017, 2017, pp. 1-5. DOI: 10.23919/SOFTCOM.2017.8115528

[19] H. Y. Adarbah, S. Ahmad, B. Arafeh, and A. Duffy, "Efficient Broadcasting for Route Discovery in Mobile Ad-hoc Networks," in 2015 International Symposium on Performance Evaluation of Computer and Telecommunication Systems (SPECTS), 2015, pp. 1-7. DOI: 10.1109/SPECTS.2015.7285290.

[20] D. G. Reina, S. L. Toral, P. Johnson, and F. Barrero, "A survey on probabilistic broadcast schemes for wireless ad hoc networks," Ad Hoc Networks, vol. 25, no. PA, pp. 263-292, 2015 doi.org/10.1016/j.adhoc.2014.10.001.

[21] P. Ruiz and P. Bouvry, "Survey on Broadcast Algorithms for Mobile Ad Hoc Networks," ACM Comput. Surv., vol. 48, no. 1, pp. 1-35, 2015. DOI:10.1145/2786005

[22] F. Palmieri, "A wave propagation-based adaptive probabilistic broadcast containment strategy for reactive MANET routing protocols," Pervasive Mob. Comput., vol. 40, pp. 628-638, 2017 doi.org/10.1016/j.pmcj.2017.06.024.

23] Q. Jiang and D. Manivannan, "Triangle-based routing for mobile ad hoc networks," Pervasive Mob. Comput., vol. 33, pp. 108-126, 2016. doi.org/10.1016/j.pmcj.2016.06.010.

[24] M. B. Khalaf, A. Y. Al-Dubai, and G. Min, "New efficient velocity-aware probabilistic route discovery schemes for high mobility Ad hoc networks," J. Comput. Syst. Sci., vol. 81, no. 1, pp. 97-109, 2015 doi.org/10.1016/j.jcss.2014.06.014.

[25] A. M. E. Ejmaa, S. Subramaniam, and Z. A. Zukarnain, "Neighbor-based Dynamic Connectivity Factor Routing Protocol for Mobile Ad Hoc Network," IEEE Access, vol. 4, pp. 8053-8064, 2016. DOI: 10.1109/ACCESS.2016.2623238

[26] M. Bani Yassein, S. F. Nimer, and A. Y. Al-Dubai, "A new dynamic counter-based broadcasting scheme for Mobile Ad hoc Networks," Simul. Model. Pract. Theory, no. 19, pp. 553-563, 2011 doi.org/10.1016/j.simpat.2010.08.011.

[27] M. B. Yassein and M. O. Khaoua, "Applications of Probabilistic Flooding in MANETs," Int. J. Ubiquitous Comput. Commun., vol. 1, no. 1, pp. 15, 2007.

[28] S. Linfoot, H. Y. Adarbah, B. Arafeh, and A. Duffy, "Impact of Physical and Virtual Carrier Sensing on the Route Discovery Mechanism in Noisy MANETs," IEEE Trans. Consum. Electron., vol. 59, no. 3, pp. 515-520, 2013. DOI: 10.1109/TCE.2013.6626232.

[29] DEI, "NS2 Library: dei80211mr," 2014. [Online]. Available: https://www.isi.edu/nsnam/ns/doc/node1.html. [Accessed: 01-Apr-2018]

[30] X. S. Rajendra and V. Boppana, "On the impact of noise on mobile ad hoc networks," in Proceedings of the 2007 international conference on Wireless communications and mobile computing (IWCMC '07)., 2007, pp. 208-213. DOI:10.1145/1280940.1280986.

[31] G. Lin, G. Noubir, and R. Rajamaran, "Mobility models for ad hoc network simulation," in Proceedings of 23rd conference of the IEEE communications society (INFOCOM 2003), 2004, pp. 454-463. DOI: 10.1109/INFCOM.2004.1354517.

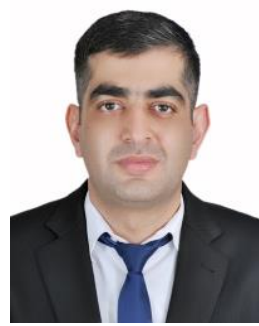

Haitham Y. Adarbah obtained his $\mathrm{PhD}$ degree in Wireless Networks from De Montfort University, Leicester, United Kingdom in 2015. He earned a Master degree in Computer Science from Amman Arab University, Amman, Jordan in 2009 and a Bachelor degree in Computer Science from AlZaytoonah University of Jordan, Amman, in 2004 $\mathrm{He}$ is an assistant professor at Gulf College, Muscat, Oman. His research interests include Route Discovery Schemes, Routing Techniques, Bandwidth Utilization, Power Consumption, Delay, Security Wireless Network, and Network Simulation \& Modelling. He is the author of more than 9 articles which were all published in various international conferences/journals.

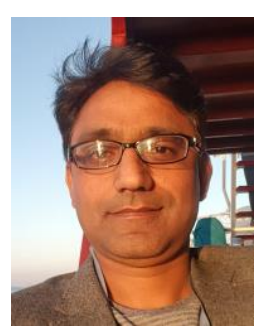

Shakeel Ahmad received the PhD (Dr.-Ing) degree from the University of Konstanz, Konstanz, Germany, in 2008 for his work on optimized network-adaptive multimedia transmission over packet erasure channels. $\mathrm{He}$ received the $\mathrm{MSc}$. degree in Information and Communication Systems from Hamburg University of Technology, Hamburg, Germany, in 2005 and the BSc. (Hons) degree in Electronics and Communication Engineering from the University of Engineering and Technology, Lahore, Pakistan, in 2000. He is an associate professor in computer networks at Southampton Solent University, UK. Shakeel Ahmad has extensive software experience in networking, communication, and information technology. His research interests include computer networks, multimedia communications, Mobile Ad-hoc Networks, Peer-to-Peer Networks, and simulation and modelling. 